\title{
Impact of healthy plant-based diet on abdominal visceral and liver fat contents
}

\author{
Lucia Pacifico ${ }^{1}$, Francesco Massimo Perla ${ }^{1}$, Claudio Chiesa ${ }^{2}$ \\ ${ }^{1}$ Department of Maternal and Child health, Sapienza University of Rome, Rome, Italy; ${ }^{2}$ Institute of Translational Pharmacology, National Research \\ Council, Rome, Italy \\ Correspondence to: Claudio Chiesa. Institute of Translational Pharmacology, National Research Council, Rome, Italy. Email: claudio.chiesa@yahoo.it. \\ Comment on: Ratjen I, Morze J, Enderle J, et al. Adherence to a plant-based diet in relation to adipose tissue volumes and liver fat content. Am J Clin \\ Nutr 2020;112:354-63.
}

Submitted Oct 15, 2020. Accepted for publication Oct 28, 2020.

doi: 10.21037/hbsn-20-760

View this article at: http://dx.doi.org/10.21037/hbsn-20-760

Diet is an important modifiable risk factor for obesity and obesity-related complications, and there has been growing interest in plant-based diets, mainly defined as "vegetarian" diets, which have been associated with a lower prevalence rate of overweight/obesity as well as a lower risk of coronary heart disease, type 2 diabetes, other cardiometabolic diseases, and all-cause mortality (1). Such dietary patterns are also a major determinant of environmental sustainability (1). Of note, however, vegetarian diets can include less healthy plant foods, such as refined grains and sugar-sweetened beverages which have detrimental effects on health (2).

In this regard, the recent development of three different plant-based diet indices has allowed an appraisal of the quality of plant-based diets: an overall plant-based diet index, reflecting a general plant-based diet; a healthy plantbased diet index, specifically based on whole grains, fruits, vegetables, legumes, nuts, tea/coffee; and an unhealthy plant-based diet index, emphasizing high intakes of refined grains, sweets, potatoes, fruit juices, and sugar-sweetened beverages (3).

Against this background, the recent article by Ratjen et al. (4) is the first to investigate the relation between consumption of different plant-based diet indices and magnetic resonance imaging (MRI) - quantified volumes of abdominal visceral adipose tissue (VAT) and subcutaneous adipose tissue (SAT) as well as of liver fat content. The study was performed in a population-based sample of 578 individuals (median age, 62 years) from Northern Germany, whose adherence to the three plant-based diet indices over the past 12 months was assessed by a validated, semiquantitative, web-based, 112-item food frequency questionnaire.

Findings from Ratjen et al. showed that adherence to a healthy plant-based diet remained associated with lower VAT, even after adjusting for several potential confounders including body mass index (BMI) (4). These cross-sectional results are somehow in line with other longitudinal studies reporting in middle-aged and elderly populations inverse associations over time between adherence to overall or specifically healthy plant-based diet and measures of adiposity such as BMI, waist circumference, fat mass index, and body fat percentage $(3,5)$. Such associations may be partly explained by the abundant intake of certain components of plant-based foods. For instance, wholegrains products and vegetables in general have low glycemic index values; vegetables are a major source of fiber, antioxidants, and chlorogenic acids; fruits are rich in fiber, antioxidants, phytochemicals, and minerals; whole grains and nuts are rich in mono- or poly-unsaturated fatty acids; and coffee and tea are rich in antioxidants and phenol chlorogenic acid. These components have been suggested to influence adiposity through their satiating effects, antiinflammatory and antioxidant properties as well as their impact on gut microbiome composition.

Considerable evidence has demonstrated that a healthy eating pattern diet across the lifespan can help reduce the risk of chronic diseases (6). Among these, nonalcoholic fatty liver disease (NAFLD) has become the most common chronic liver disease worldwide. Prior to the 
article by Ratjen et al. (4), only one study sought to shed light on the relationship between adherence to plantbased diets and likelihood of fatty liver (7). In that study, Mazidi et al. reported an inverse association between a healthy plant-based diet and NAFLD risk based on fatty liver index (7). This steatosis biomarker has shown an overall good diagnostic performance in several population studies; however, it has only been validated against liver ultrasonography (8). Conversely, Ratjen et al. failed to find convincing evidence of associations of the different plant-based diet indices with liver fat, raising a general note of caution for future studies on the topic. Unlike the previous study, it is worth noticing that Ratjen et al. not only excluded secondary casues for liver fat accumulation, but also took an important step to quantify the hepatic fat content by using the MRI technique. While other modalities such as ultrasound and computed tomography are capable of providing only qualitative information about hepatic steatosis, MRI represents a validated non-invasive means to accurately quantify intrahepatic lipid content (9).

The relationship between diet and development of NAFLD is complex. Though the most studied protective dietary pattern in NAFLD is the Mediterranean diet, available evidence for recommending Mediterranean diet against NAFLD is of moderate quality $(10,11)$. Yet, studies assessing the role of "vegetarian diets" against NAFLD have yielded conflicting results (7). Accordingly, current findings from Ratjen et al. (4) have the potential to steer further research on the impact of plant-based diet on NAFLD as a heterogeneous disease. It is well-documented that NAFLD comprises a wide spectrum of liver histologic abnormalities ranging from a simple fat accumulation to advanced forms of liver involvement with a necro-inflammatory response catalysing the progression from NAFLD to nonalcoholic steatohepatitis (NASH). This disease spectrum has led to the formulation of the multiple-hit hypothesis, where a subclinical pathology such as a lipid accumulation in hepatocytes can make the liver more vulnerable to parallel hits stemming from localized oxidative stress and proinflammatory mediators derived from peripheral tissues (12), and acting in concert to regulate the progression of the disease to NASH. Therefore, though findings by Ratjen et al. display no inverse association between a healthy plant-based diet and liver fat content (4), we cannot rule out the possibility that adherence to a healthy plant-rich dietary pattern might infer a benefit in NAFLD patients thanks to its anti-inflammatory and antioxidant effects on the noxa accounting for severity and progression of liver damage. In this context, more insight into the short-and long-term effects of such dietary pattern on histologic liver outcomes of NAFLD patients should be performed. Parallel efforts should be made to optimize adherence. In that vein, in order to ensure accountability of future dietary interventions in this area, a genetic analysis should also be considered to substantiate responders and non-responders to various dietary patterns including plantbased diets (13).

Although an unhealthy diet is a typical risk factor for NAFLD, of great interest is also the interplay between diet and gut microbiota which can play a crucial role in the development and progression of NAFLD $(14,15)$. Recent advances in the field have suggested that increased intake of obesogenic foods may affect the composition and diversity of the gut microbiota, as well as the intestinal barrier and the gut vascular barrier leading to increased gut permeability to bacteria and bacteria-derived products, which, in turn, might result in metabolic endotoxemia and low-grade inflammation, hence contributing to the development of obesity-associated fatty liver disease/NASH (14). Thus, to provide a better understanding of the impact of distinct dietary patterns including plant-based diets on NAFLD/ NASH patients, future studies are needed to explore in such patients the mutualism between diet and its gut commensal microbiota, in particolar which dietary factors affect gut microbiota, how they do so, and the changes in microbial metabolic activity as a result of the dietary composition (14). In this respect, ageing and genetics might also have an important role in the interplay between diet and constitutional profiles of gut microbiota (15).

\section{Acknowledgments}

Funding: None.

\section{Footnote}

Provenance and Peer Review: This article was commissioned by the editorial office, Hepatobiliary Surgery and Nutrition. The article did not undergo external peer review.

Conflicts of Interest: All authors have completed the ICMJE uniform disclosure form (available at https://hbsn. amegroups.com/article/view/10.21037/hbsn-20-760/coif). The authors have no conflicts of interest to declare.

Ethical Statement: The authors are accountable for all 
aspects of the work in ensuring that questions related to the accuracy or integrity of any part of the work are appropriately investigated and resolved.

Open Access Statement: This is an Open Access article distributed in accordance with the Creative Commons Attribution-NonCommercial-NoDerivs 4.0 International License (CC BY-NC-ND 4.0), which permits the noncommercial replication and distribution of the article with the strict proviso that no changes or edits are made and the original work is properly cited (including links to both the formal publication through the relevant DOI and the license). See: https://creativecommons.org/licenses/by-nc-nd/4.0/.

\section{References}

1. Magkos F, Tetens I, Bügel SG, et al. A perspective on the transition to plant-based diets: a diet change may attenuate climate change, but can it also attenuate obesity and chronic disease risk? Adv Nutr 2020;11:1-9.

2. Hemler EC, Hu FB. Plant-Based Diets for Cardiovascular Disease Prevention: All Plant Foods Are Not Created Equal. Curr Atheroscler Rep 2019;21:18.

3. Satija A, Malik V, Rimm EB, et al. Changes in intake of plant-based diets and weight change: results from 3 prospective cohort studies. Am J Clin Nutr 2019;110:574-82.

4. Ratjen I, Morze J, Enderle J, et al. Adherence to a plantbased diet in relation to adipose tissue volumes and liver fat content. Am J Clin Nutr 2020;112:354-63.

5. Chen Z, Schoufour JD, Rivadeneira F, et al. Plantbased diet and adiposity over time in a middle-aged and elderly population: the rotterdam study. Epidemiology 2019;30:303-10.

6. US Department of Health and Human Services; US

Cite this article as: Pacifico L, Perla FM, Chiesa C. Impact of healthy plant-based diet on abdominal visceral and liver fat contents. HepatoBiliary Surg Nutr 2021;10(1):136-138. doi: 10.21037/hbsn-20-760
Department of Agriculture. Dietary Guidelines for Americans: 2015-2020. 8th ed. December 2015. Accessed October 15, 2020. Available online: https://health.gov/ dietaryguidelines/2015/guidelines/

7. Mazidi M, Kengne AP. Higher adherence to plant-based diets are associated with lower likelihood of fatty liver. Clin Nutr 2019;38:1672-7.

8. Fedchuk L, Nascimbeni F, Pais R, et al. Performance and limitations of steatosis biomarkers in patients with nonalcoholic fatty liver disease. Aliment Pharmacol Ther 2014;40:1209-22.

9. Springer F, Machann J, Claussen CD, et al. Liver fat content determined by magnetic resonance imaging and spectroscopy. World J Gastroenterol 2010;16:1560-6.

10. Perdomo CM, Frühbeck G, Escalada J. Impact of nutritional changes on nonalcoholic fatty liver disease. Nutrients 2019;11:677.

11. Anania C, Perla FM, Olivero F, et al. Mediterranean diet and nonalcoholic fatty liver disease. World J Gastroenterol 2018;24:2083-94.

12. Riazi K, Raman M, Taylor L, et al. Dietary Patterns and Components in Nonalcoholic Fatty Liver Disease (NAFLD): What Key Messages Can Health Care Providers Offer? Nutrients 2019;11:2878.

13. George ES, Forsyth A, Itsiopoulos C, et al. Practical Dietary Recommendations for the Prevention and Management of Nonalcoholic Fatty Liver Disease in Adults. Adv Nutr 2018;9:30-40.

14. Kirpich IA, Marsano LS, McClain CJ. Gut-liver axis, nutrition, and non-alcoholic fatty liver disease. Clin Biochem 2015;48:923-30.

15. Yu J, Marsh S, Hu J, et al. The Pathogenesis of Nonalcoholic Fatty Liver Disease: Interplay between Diet, Gut Microbiota, and Genetic Background. Gastroenterol Res Pract 2016;2016:2862173. 